\title{
Communication \\ Pre-Chirp-Managed Adiabatic Soliton Compression in Pressure-Gradient Hollow-Core Fibers
}

\author{
Runzhi Chen ${ }^{1,2}$, Zhuo Shi ${ }^{1,2}$ and Guoqing Chang 1,2,3,* \\ 1 Beijing National Laboratory for Condensed Matter Physics, Institute of Physics, Chinese Academy \\ of Sciences, Beijing 100190, China; runzhi.chen@iphy.ac.cn (R.C.); shizhuo20@mails.ucas.ac.cn (Z.S.) \\ 2 University of Chinese Academy of Sciences, Beijing 100049, China \\ 3 Songshan Lake Materials Laboratory, Dongguan 523808, China \\ * Correspondence: guoqing.chang@iphy.ac.cn
}

\section{check for} updates

Citation: Chen, R.; Shi, Z.; Chang, G. Pre-Chirp-Managed Adiabatic Soliton Compression in Pressure-Gradient Hollow-Core Fibers. Photonics 2021, 8, 357. https://doi.org/10.3390/ photonics 8090357

Received: 4 July 2021

Accepted: 23 August 2021

Published: 27 August 2021

Publisher's Note: MDPI stays neutral with regard to jurisdictional claims in published maps and institutional affiliations.

Copyright: (c) 2021 by the authors. Licensee MDPI, Basel, Switzerland. This article is an open access article distributed under the terms and conditions of the Creative Commons Attribution (CC BY) license (https:// creativecommons.org/licenses/by/ $4.0 /)$.

\begin{abstract}
Post-pulse-compression is demanded to produce energetic few-cycle pulses. We propose pre-chirp-managed adiabatic soliton compression (ASC) in gas-filled pressure-gradient hollow-core fibers to suppress the detrimental pedestals and therefore significantly improve the compressed pulse quality. We show that two-stage ASC can compress $125 \mu \mathrm{J}, 130 \mathrm{fs}$ pulses at $2 \mu \mathrm{m}$ to a nearly two-cycle pulse $15 \mathrm{fs}$ in duration. Our analytical analysis suggests that ASC is in favor of compressing pulses centered at a longer wavelength. As an example, a $280 \mu \mathrm{J}, 220 \mathrm{fs}$ Gaussian pulse at $4 \mu \mathrm{m}$ is compressed to $60 \mathrm{fs}$ with minimal pedestals. We expect that the resulting high-quality, energetic few-cycle pulses will find important applications in high-field science.
\end{abstract}

Keywords: adiabatic soliton compression; pressure gradient; hollow-core fibers

\section{Introduction}

High-energy $(>100 \mu \mathrm{J})$ few-cycle optical pulses with high average power $(>100 \mathrm{~W})$ are increasingly demanded by high-field science. To date, nearly all these powerful and energetic pulses are obtained via post-compression of relatively long pulses [1]. In general, pulse compression techniques can be divided into two types: (1) nonlinear spectral broadening followed by a dispersive device for proper phase compensation and (2) soliton self-compression without further phase removal. Both types of compression involve strong nonlinear light-matter interaction such that the optical pulse acquires a huge nonlinear phase, resulting in substantial spectral broadening. In the first type of pulse compression (e.g., multi-plate compression), the spectrally broadened pulse develops frequency chirp and thus requires dispersive devices such as chirped mirrors for pulse dechirping to generate a few-cycle pulse. In contrast, the second type of compression relies on the formation of a high-order soliton as a result of pulse propagation in a waveguide with Kerr nonlinearity and negative group-velocity dispersion (GVD). More specifically, an input pulse given enough energy evolves into a high-order soliton inside the waveguide and experiences spectral broadening. Such evolution leads to a compressed, chirp-free pulse, which does not need an extra dechirping device. Although both types of pulse compression techniques are successful in producing high-power, energetic few-cycle pulses, they suffer one common drawback: the compressed pulse exhibits a relatively large pedestal, which compromises its applications in high-field science. This unwanted pedestal in the first type of compression arises from the uncompensated high-order spectral phase; for the second type, it originates from the intrinsic property of a high-order soliton.

Besides high-order soliton compression, compressed chirp-free pulses can also be obtained using adiabatic soliton compression (ASC) [2-13]. ASC differs from high-order soliton compression in that the pulse to be compressed evolves into a fundamental soliton as propagating in a waveguide with negative GVD. A fundamental soliton results from a balance between Kerr nonlinearity and GVD, and hence increasing the nonlinearity or 
decreasing the GVD along the pulse propagation renders a gradual decrease in the pulse duration to reinforce a local nonlinearity-GVD balance [2]. Consequently, the input pulse is continuously compressed along the propagation. Despite the perturbation by high-order nonlinearities and dispersion, the compressed pulse stays close to be a fundamental soliton, and the pedestal is significantly suppressed.

ASC has been demonstrated to achieve pulse compression in single-mode fibers with amplification [3-5], dispersion-decreasing fibers [6-9], and non-uniform grating structures [10]. All these demonstrations were achieved in fibers with solid cores. Recent years have seen intense research on nonlinear optical phenomena arising from propagation of femtosecond pulses inside gas-filled hollow-core fibers (HCFs) [11]. These HCFs are widely used to implement the above-mentioned two types of pulse compression [1]. However, ASC in gas-filled hollow-core fibers is rarely investigated. Lægsgaard et al. presented a theoretical study on ASC limited by third-order dispersion and the Raman effect in photonic bandgap fibers [12]. Huang et al. experimentally studied ASC in the presence of strong ionization, which demonstrated a $300 \mathrm{~nm}$ blueshift of the soliton central wavelength [13]. In this paper, we study the ASC of femtosecond pulses in pressure-gradient HCFs, with the focus on how to optimize the process to produce high-quality, pedestal-free few-cycle pulses. We show that introducing positive pre-chirp to the pulse prior to compression facilitates ASC and improves the compressed pulse quality. The pedestal stays $\sim 30 \mathrm{~dB}$ below the pulse peak. We further demonstrate that two-stage ASC can generate two-cycle pulses at $2 \mu \mathrm{m}$. We also discuss the performance of ASC in compressing pulses centered at $4 \mu \mathrm{m}$.

\section{Simple Analysis of ASC in Pressure-Gradient HCFs Based on the Soliton Area Theorem}

According to Ref. [14], the GVD of the fundamental mode in an HCF is given by

$$
\beta_{2}=\frac{\rho_{r}}{c} f(\omega)-\frac{c u_{01}^{2}}{\omega^{3} a^{2}}
$$

where $\rho_{r}$ is the gas density relative to standard conditions, $c$ the speed of light in vacuum, $u_{01}$ the first zero of the zeroth-order Bessel function of the first kind, and $a$ the core radius. $f(\omega)=\partial_{\omega} \chi^{(1)}+\omega \partial_{\omega}^{2} \chi^{(1)} / 2$, and $\partial_{\omega}$ is the derivative with respect to the frequency $\omega$. $\chi^{(1)}(\omega)$ is the linear susceptibility of the gas. Equation (1) shows that GVD includes two contributions: (1) positive gas dispersion and (2) negative waveguide dispersion.

The Kerr nonlinear parameter of the gas is defined by $\gamma=n_{2} \omega_{0} /\left(c A_{e f f}\right)$, where $n_{2}$ is the nonlinear index coefficient. $A_{e f f} \approx 1.65 a^{2}$ is the effective mode area for a negativecurvature HCF (e.g., antiresonant HCF) [15]. The nonlinear parameter is related to the gas density and fiber core radius by

$$
\gamma=\frac{n_{2} \omega_{0}}{c A_{e f f}}=\frac{n_{20} \omega_{0} \rho_{r}}{1.65 a^{2} c}
$$

where $n_{20}$ corresponds to the nonlinear index coefficient of the gas under standard conditions. The well-known soliton area theorem connects the pulse energy $E_{0}$ and the full-width at half-maximum (FWHM) of the fundamental soliton, $T_{0}$, written as

$$
\frac{\gamma E_{0} T_{0}}{3.5\left|\beta_{2}\left(\omega_{0}\right)\right|}=1 \text {. }
$$

Plugging Equations (1) and (2) into Equation (3) and considering $\rho_{r}$ being proportional to pressure $p$, we obtain the following equation:

$$
E_{0} T_{0}=\frac{3.5\left|\beta_{2}\left(\omega_{0}\right)\right|}{\gamma} \approx \frac{5.8 u_{01}^{2} c^{2}}{\omega_{0}^{4} n_{20} \rho_{r}}-\frac{5.8 f\left(\omega_{0}\right) a^{2}}{\omega_{0} n_{20}}=\frac{A_{1}}{p}-A_{2} a^{2} .
$$


$A_{1}$ and $A_{2}$ are constants given by $A_{1}=\frac{5.8 u_{01}^{2} c^{2} p_{a t m}}{\omega_{0}^{4} n_{20}}$ and $A_{2}=\frac{5.8 f\left(\omega_{0}\right)}{\omega_{0} n_{20}} . p_{a t m}$ is set at $1 \mathrm{~atm}$. Equation (4) clearly shows that increasing the gas pressure along an HCF can effectively decrease the pulse duration. Indeed, pressure-gradient HCFs are widely employed to control the nonlinear interaction between the filled noble gas and the optical pulse [16-18]. Equation (4) seems to suggest that increasing the input pulse energy is another efficient way of shorting the pulse duration. However, an increased pulse energy and reduced duration may result in high enough peak power that causes strong ionization and therefore degrades the compressed pulse quality. To avoid detrimental ionization effects, we set a limit to the gas ionization such that the variation of the refractive index induced by gas ionization is less than the index variation induced by the Kerr effect. Such a limit leads to following equation that connects the pulse energy, the pulse duration, and the fiber core radius [19]:

$$
a \geq A_{3} T_{0}^{-\alpha} E_{0}^{\beta}
$$

where $\alpha \simeq 0.45$ and $\beta \simeq 0.51 . A_{3}$ is a constant, which depends on the filled gas.

A combination of Equations (4) and (5) determines the maximum input pulse energy and the minimum pulse duration at the output of the pressure-gradient HCF:

$$
\begin{aligned}
E_{\text {max }} & =\left[\frac{a}{A_{3}}\left(\frac{A_{1}}{p_{\text {out }}}-A_{2} a^{2}\right)^{\alpha}\right]^{\frac{1}{\alpha+\beta}} \\
T_{\text {min }} & =\left[\frac{A_{3}}{a}\left(\frac{A_{1}}{p_{\text {out }}}-A_{2} a^{2}\right)^{\beta}\right]^{\frac{1}{\alpha+\beta}}
\end{aligned}
$$

$p_{\text {out }}$ is the gas pressure at the fiber output. In most cases, the gas dispersion is much smaller than the waveguide dispersion, and therefore Equations (6) and (7) are further simplified as

$$
\begin{aligned}
& E_{\text {max }}=\left[\frac{a}{A_{3}}\left(\frac{A_{1}}{p_{\text {out }}}\right)^{\alpha}\right]^{\frac{1}{\alpha+\beta}} \\
& T_{\text {min }}=\left[\frac{A_{3}}{a}\left(\frac{A_{1}}{p_{\text {out }}}\right)^{\beta}\right]^{\frac{1}{\alpha+\beta}}
\end{aligned}
$$

Figure 1 shows the dependence of $E_{\max }$ and $T_{\min }$ on the HCF core radius at different gas pressure values. We assume the HCF is filled with helium and the pulse is centered at $2 \mu \mathrm{m}$, which leads to $A_{3} \approx 12.6 \mu \mathrm{m} \mathrm{fs}{ }^{\alpha} / \mu \mathrm{J}^{\beta}$. Given that the helium pressure at the HCF output is fixed, $E_{\max }$ increases and $T_{\min }$ decreases as the fiber core radius is increased. At a fixed fiber core radius, increasing the helium pressure reduces both $E_{\max }$ and $T_{\min }$.

Recent years have seen a huge effort to develop high-energy few-cycle pulses with the center wavelength at $\sim 4 \mu \mathrm{m}$, aiming for driving high-harmonic generation to extend the cut-off energy and produce $\mathrm{keV}$ photons $[20,21]$. These pulses were obtained via postcompression of initial 100 -fs pulses delivered by optical parametric amplifiers. Indeed, ASC in pressure-gradient HCFs is in favor of compressing high-energy pulses at a longer wavelength. Besides the fiber core radius and gas pressure, two other parameters, $A_{1}$ and $A_{3}$, appear in Equations (8) and (9). $A_{3}$ is weakly dependent on the pulse wavelength, and it is $13.9 \mu \mathrm{m} \mathrm{fs}{ }^{\alpha} / \mu \mathrm{J}^{\beta}$ at $4 \mu \mathrm{m}$. However, $A_{1}$ is proportional to the fourth power of the wavelength. As Equation (8) shows, increasing the center wavelength from $2 \mu \mathrm{m}$ to $4 \mu \mathrm{m}$ can increase $E_{\max }$ by a factor of $\sim 4$. Figure 2 shows the dependence of $E_{\max }$ and $T_{\min }$ on the core radius of an $\mathrm{HCF}$ at different helium pressure values for the pulse centered at $4 \mu \mathrm{m}$. The results indicate that ASC in pressure-gradient HCFs is able to compress sub-mJ pulses at $4 \mu \mathrm{m}$. 

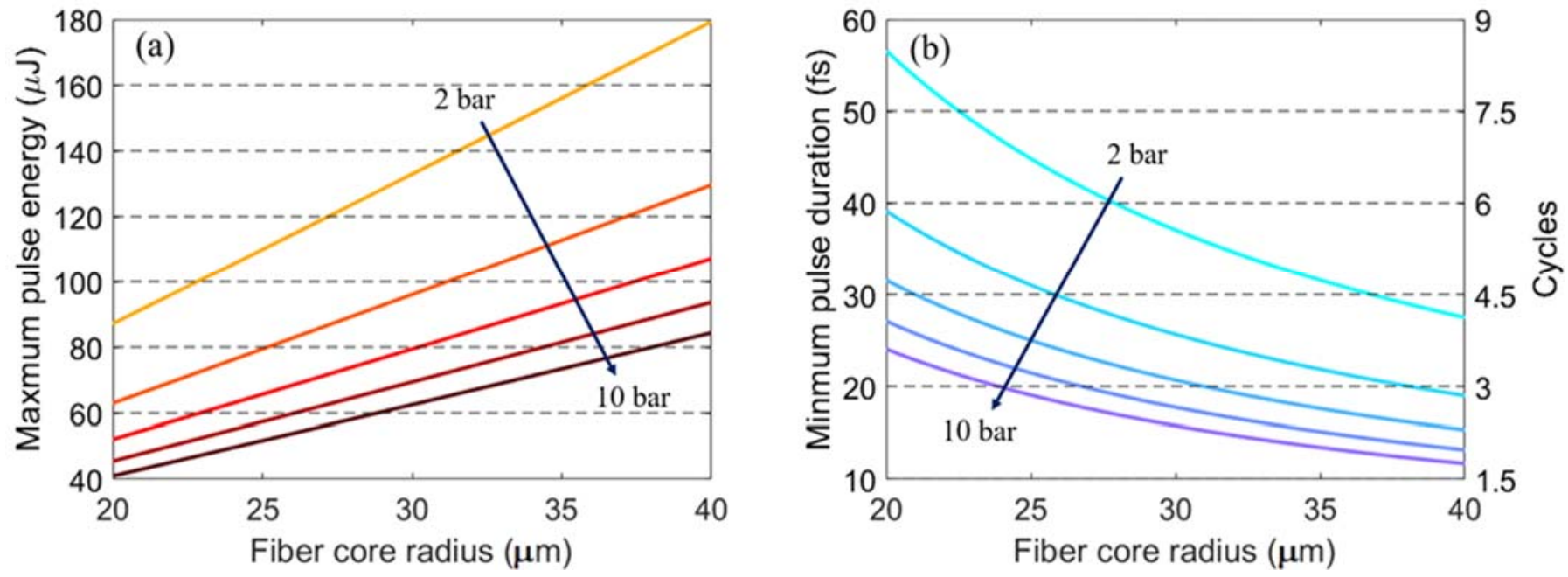

Figure 1. Dependence of $E_{\max }(\mathbf{a})$ and $T_{\min }(\mathbf{b})$ on the HCF core radius with the helium pressure at 2 bar, 4 bar, 6 bar, 8 bar, and 10 bar. The pulse is centered at $2 \mu \mathrm{m}$.
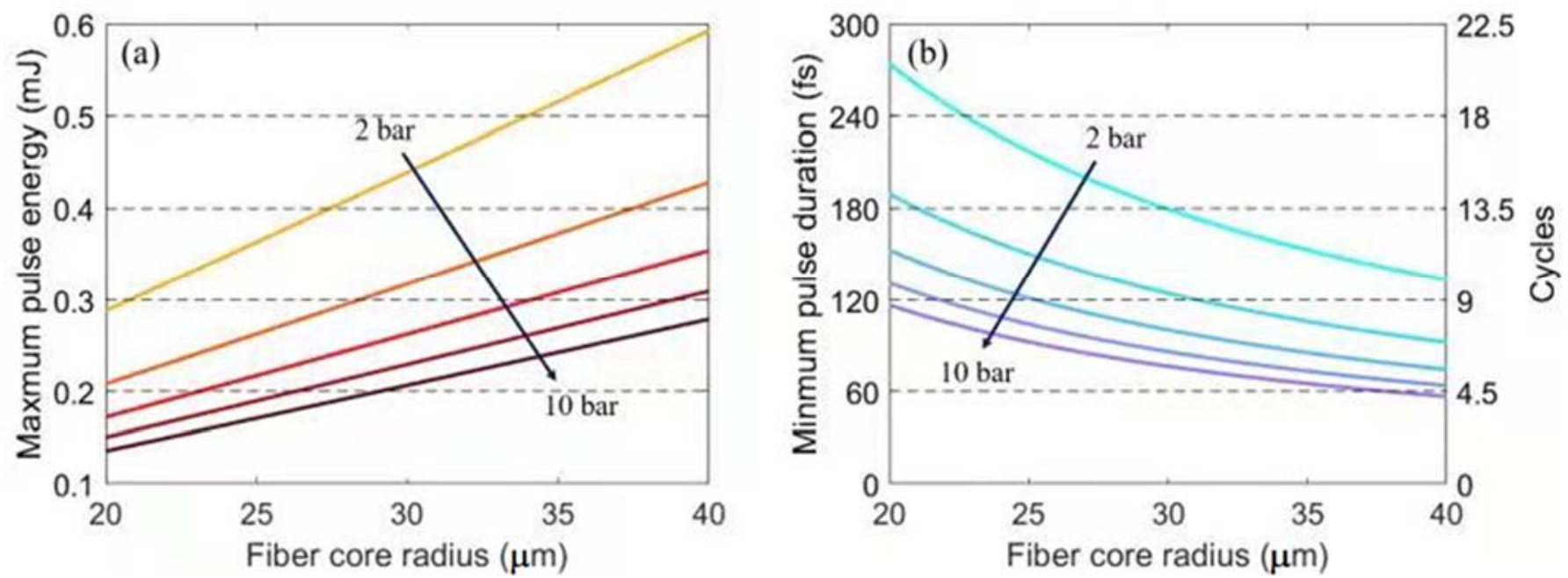

Figure 2. Dependence of $E_{\max }(\mathbf{a})$ and $T_{\min }(\mathbf{b})$ on the HCF core radius with the helium pressure at 2 bar, 4 bar, 6 bar, 8 bar, and 10 bar. The pulse is centered at $4 \mu \mathrm{m}$.

\section{Simulation Results and Discussion}

Although Equations (8) and (9) predict the maximum pulse energy and the minimum pulse duration as a function of the HCF radius and the filled gas pressure, they provide no information about the compressed pulse quality. To further investigate the ASC process in detail, we solve the generalized nonlinear Schrödinger equation (GNLSE), taking into account dispersion, Kerr nonlinearity, and ionization effects [22]:

$$
\partial_{z} \psi=i\left[\sum_{m \geq 2} \frac{\beta_{m}\left(i \partial_{t}\right)^{m}}{m !}+\gamma\left(1+i \omega_{0}^{-1} \partial_{t}\right)|\psi(t)|^{2}-\frac{\omega_{p}^{2}}{2 \omega_{0} c}+i \frac{A_{e f f} I_{p} \partial_{t} N_{e}}{2|\psi(z, t)|^{2}}\right] \psi,
$$

where $\psi$ corresponds to the complex field envelope of the pulse and $\beta_{m}$ denotes the $m^{\text {th }}$-order dispersion coefficient calculated at the center frequency $\omega_{0} . I_{p}, N_{e}$, and $A_{e f f}$ represent the gas ionization energy, the free-electron density, and the effective mode area, respectively. The plasma frequency $\omega_{p}$ is given by $\omega_{p}=\left[e^{2} N_{e} /\left(\varepsilon_{0} m_{e}\right)\right]^{0.5}$, where $\varepsilon_{0}$ is the 
vacuum permittivity, $e$ the electronic charge, and $m_{e}$ the electron mass. The free electron density $N_{e}$ is given by [23]:

$$
\frac{\partial N_{e}}{\partial t}=W(t)\left(N_{0}-N_{e}\right),
$$

where $W(t)$ is the ionization rate and $N_{0}$ is the density of gas atoms. The effects of electron attachment and recombination are neglected in Equation (2) for pulses shorter than 1 ps. In this letter, we use Ammosov-Delone-Krainov (ADK) theory to calculate the ionization rate $W(t)[24]$ :

$$
W(t)=\omega_{t r}\left|C_{n^{*}}\right|^{2}\left(\frac{4 \omega_{t r}}{\omega_{t}}\right)^{2 n^{*}-1} \exp \left(-\frac{4 \omega_{t r}}{3 \omega_{t}}\right)
$$

where $\omega_{t r}=I_{p} / \hbar$ is the transition frequency and $n^{*}=Z\left(I_{p h} / I_{p}\right)^{0.5}$ is the effective principal quantum number. $\omega_{t}=e|E(z, t)|\left[2 m_{e} I_{p}\right]^{-0.5}$ is the tunneling frequency, in which the carrier-resolved electric field $E(z, t)$ is given by $E(z, t)=\sqrt{2 / \varepsilon_{0} n_{0} c A_{e f f}} \psi(z, t)$, and $n_{0}$ is the linear refractive index. $\left|C_{n^{*}}\right|^{2}=2^{2 n^{*}}\left[n^{*} \Gamma\left(n^{*}+1\right) \Gamma\left(n^{*}\right)\right]^{-1}$, where $Z$ is the net resulting charge of the ion, $I_{p h}$ is the ionization energy of the hydrogen atom, and $\Gamma(x)$ is the Euler gamma function. It is noteworthy that Equation (10) remains valid even as the pulse duration approaches one carrier oscillation cycle [25].

For a pressure-gradient $\mathrm{HCF}$, the gas pressure along the fiber is given by

$$
p(z)^{2}=p_{0}^{2}+\frac{z}{L}\left(p_{L}^{2}-p_{0}^{2}\right) .
$$

$p_{0}$ and $p_{L}$ denote the pressure at the fiber input end and the output end, respectively. $L$ represents the fiber length.

In the first simulation, we investigate the ASC of pulses at $2 \mu \mathrm{m}$ in antiresonant $\mathrm{HCFs}$, which feature a large mode area, low transmission loss, negative dispersion, and broadband transmission [26]. We assume that the antiresonant HCF is $8 \mathrm{~m}$ long with a core radius of $28 \mu \mathrm{m}$ filled with helium with the pressure varying from $p_{0}=0.6$ bar to $p_{L}=2.6$ bar. The input pulse is a transform-limited Gaussian pulse centered at $2 \mu \mathrm{m}$ with $100 \mu \mathrm{J}$ pulse energy and $110 \mathrm{fs}$ duration. These pulses can be experimentally obtained by state-of-the-art high-power Tm-fiber amplifier systems [18].

Figure 3a shows the pulse evolution along the fiber in the logarithmic scale. Due to increased gas pressure leading to a reduced group velocity, the center of gravity of the pulse shifts toward a later time. The central part of the pulse becomes narrower continuously during the propagation of the first $5 \mathrm{~m}$; for further propagation, it becomes wider and then narrower again. During the propagation, the ionization is minimal, as expected. The black curve in Figure $3 \mathrm{~b}$ plots the FWHM of the pulse as a function of the propagation distance, and the blue curve shows the FWHM of the corresponding fundamental soliton that shares the same energy and nonlinear coefficient as the pulse propagating in the HCF. Clearly, the pulse duration oscillates around the fundamental soliton duration, indicating that the pulse adiabatically evolves into a fundamental soliton.

The quality of a pulse is quantified by the Strehl ratio defined by the peak-power ratio between the compressed pulse and the transform-limited pulse. A pulse with a Strehl ratio of $\sim 0.8$ is regarded as a decent pulse. However, most high-energy pulses obtained by post-compression exhibit much lower Strehl ratios, with a large portion of energy shed in pedestals and satellite pulses. Figure $3 \mathrm{c}$ plots the output pulse (black curve), the transform-limited pulse calculated from the output spectrum (red dashed curve), and the fundamental soliton determined by the dispersion/nonlinearity at the fiber output (blue curve). The input $110 \mathrm{fs}$ pulse is compressed to $38 \mathrm{fs}$, which is close to the transform-limited pulse with the Strehl ratio reaching as high as $\sim 0.99$. The pedestal stays $>28 \mathrm{~dB}$ below the pulse peak; the central part of the compressed pulse has an excellent overlap with the fundamental soliton pulse and contains $97.5 \%$ of the total pulse energy. 
The results in Figure $3 b$ shows that the pulse evolves toward a fundamental soliton. However, the output spectrum (Figure 3d) clearly indicates that the compressed pulse deviates much from an ideal fundamental soliton that features a spectrum of hyperbolic secant shape. It is well known that pre-chirping the input pulse into an optical fiber can be used to control the nonlinear propagation. The pre-chirp management technique has been applied to nonlinear pulse amplification $[27,28]$, delivery of femtosecond pulses [29-31], nonlinear pulse compression [32-34], dispersive wave generation [35], soliton self-frequency shift [36], and self-phase modulation enabled spectral selection for generating widely tunable femtosecond pulses [37]. Here we propose to pre-chirp the input pulse to control ASC in pressure-gradient HCFs. Figure 4 shows the simulation results corresponding to the ASC of a positively chirped pulse. In the simulation, we added $2000 \mathrm{fs}^{2}$ group-delay dispersion (GDD) to positively pre-chirp the $100 \mu \mathrm{J}, 110 \mathrm{fs}$ Gaussian pulse to a duration of $120 \mathrm{fs}$. All other parameters remained unchanged. Figure 4a plots the pulse evolution. A comparison between Figures $3 \mathrm{a}$ and $4 \mathrm{a}$ shows that introducing pre-chirp reduces the duration oscillation of the central part in the compressed pulse. This is more evidently revealed by the results in Figure 4b: due to the negative GVD, the positively chirped pulse is shortened more rapidly at the beginning of the compression; the FWHM of the compressed pulse quickly converges to the FWHM of the fundamental soliton after $3 \mathrm{~m}$ propagation. At the HCF output, the optical spectrum (black curve in Figure 4d) is close to the soliton spectrum (blue curve in Figure 4d). Consequently, compared with the ASC of the un-chirped pulse, pre-chirp-managed ASC leads to a compressed pulse with the pedestal further suppressed (Figure 4c). In this case, the pedestal stays $>37 \mathrm{~dB}$ below the pulse peak. The input pre-chirped $120 \mathrm{fs}$ pulse is compressed to $37 \mathrm{fs}$ (corresponding to 5.5 cycles) with the Strehl ratio reaching as high as $\sim 0.99$, and the central part of the compressed pulse contains $99 \%$ of the total pulse energy.

The results in Figure 4 demonstrate that pre-chirp-managed ASC in pressure-gradient HCFs can produce high-quality few-cycle pulses with the pedestal significantly suppressed. However, our extensive simulation shows that the compression ratio is limited to 3-4 to suppress the pedestal by more than $30 \mathrm{~dB}$. This is caused by the characteristic pressure gradient determined by Equation (10): the square of the gas pressure increases linearly with the fiber length, and accordingly the adiabatic soliton duration drops sharply at the beginning of the HCF and then quickly flattens out. It is hard (if not impossible) to achieve a higher compression ratio via just pre-chirping the input pulse to reinforce the ASC. This drawback of low compression ratio can be alleviated by cascading the ASC in multiple stages.

The results in Figure 5 show two-stage pre-chirp-managed ASC that compresses an initial unchirped $125 \mu \mathrm{J}, 130 \mathrm{fs}$ pulse down to $15 \mathrm{fs}$ in duration. In the first stage, the 5 -m-long HCF has a core radius of $30 \mu \mathrm{m}$, and the helium pressure varies from $p_{0}=0.2 \mathrm{bar}$ to $p_{L}=1.4 \mathrm{bar}$. The 5-m-long HCF in the second stage has a core radius of $45 \mu \mathrm{m}$, and the helium pressure varies from $p_{0}=0.5$ bar to $p_{L}=6$ bar. We assume a coupling loss of $20 \%$ such that the pulse energy is reduced to $100 \mu \mathrm{J}$ at the input of the second HCF. Given the core difference ( $30 \mu \mathrm{m}$ versus $45 \mu \mathrm{m}$ ) between the two HCFs, the first fiber has a GVD about 2.25 times larger than the second one, since gas dispersion is much smaller compared with waveguide dispersion. As the results in Refs. [38,39] have shown, a soliton experiencing an abrupt dispersion change may cause dispersive shedding and soliton degradation. Such a detrimental effect can be mitigated by introducing a finite-length fiber junction between the two fibers with a large dispersion difference $[38,39]$. In our situation, instead of adding a fiber junction, we properly pre-chirp the soliton before entering the second HCF to ensure ASC. More specifically, we add $2500 \mathrm{fs}^{2}\left(500 \mathrm{fs}^{2}\right)$ GDD to the pulse at the input of the first (second) HCF. The input pulse is compressed to $54 \mathrm{fs}$ inside the first HCF and then compressed to 15 fs by the second HCF corresponding to 2.3 optical cycles.

It is noteworthy that using gas-filled HCFs to self-compress $2 \mu \mathrm{m}$ pulses to fewcycle duration has been experimentally demonstrated by several groups $[18,40]$. The selfcompression was based on high-order soliton compression, and therefore the compressed 
pulse quality was compromised. For example, Gebhardt et al. employed a $42 \mathrm{~cm}$ gasfilled antiresonant HCF to compress $34.4 \mu \mathrm{J}$ pulses centered at $1.8 \mu \mathrm{m}$ from $110 \mathrm{fs}$ to $13 \mathrm{fs}$. However, the compressed 2.1-cycle pulse has elongated pedestals that are less than $10 \mathrm{~dB}$ below the main peak [18]. Assisted by numerical simulation, the authors estimated that the central part of the pulse only includes $60 \%$ of the pulse energy [18]. In contrast, our results in Figure 5 show that two-stage pre-chirp-managed ASC in pressure-gradient HCFs can generate nearly pedestal-free nearly two-cycle pulses with $\sim 98 \%$ energy confined in the central part of the compressed pulse.
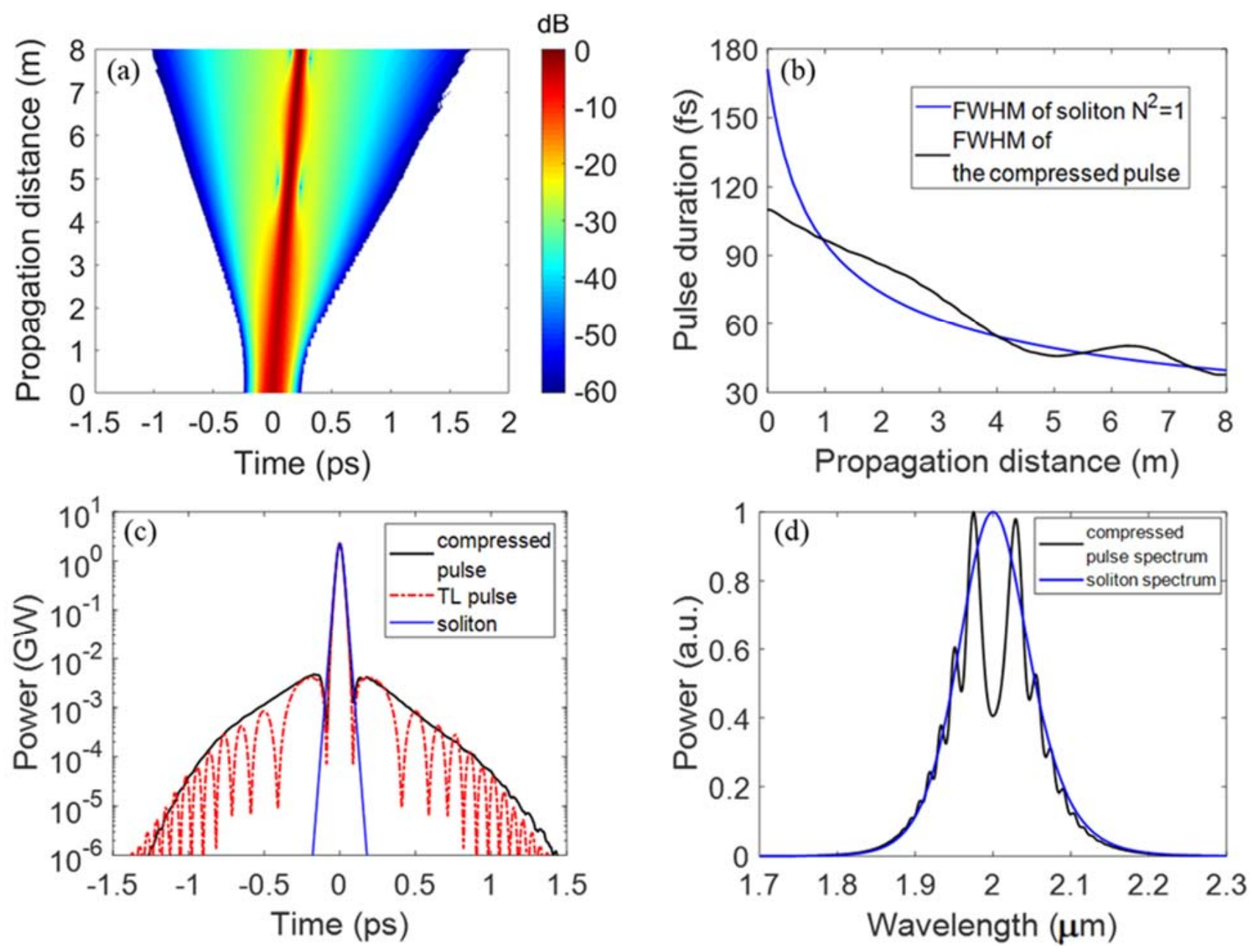

Figure 3. ASC of an unchirped $110 \mathrm{fs}, 100 \mu \mathrm{J}$ pulse at $2 \mu \mathrm{m}$. (a) Pulse evolution as a function of propagation distance. The intensity is shown in log scale. (b) FWHM of a fundamental soliton (blue curve) and FWHM of a compressed pulse (black curve) versus the propagation distance. (c) Compressed pulse (black curve) and transform-limited (TL) pulse (red dashed curve) at HCF output. The fundamental soliton is shown as the blue curve. (d) Optical spectrum (black curve) and soliton spectrum (blue curve) at HCF output. 

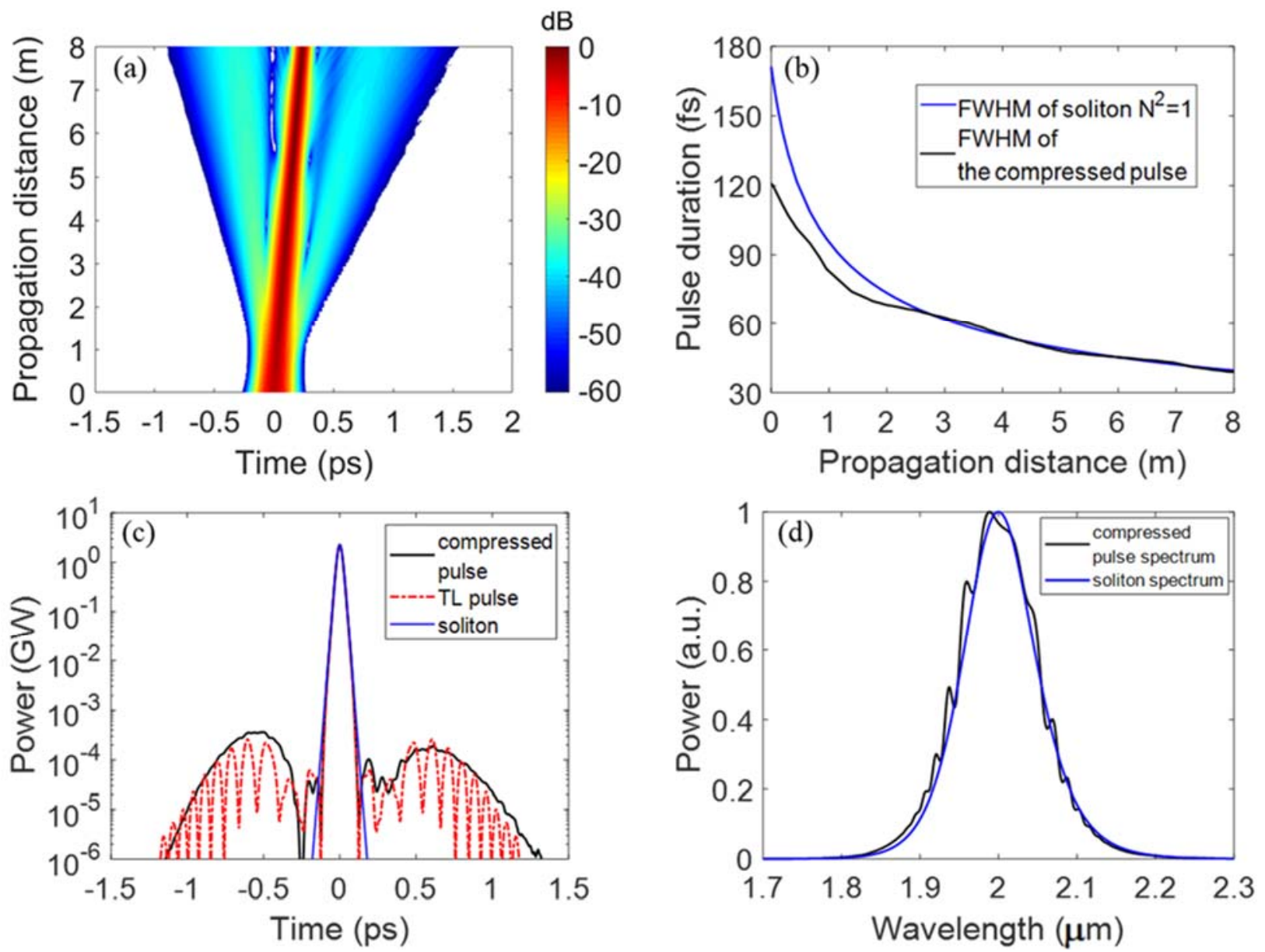

Figure 4. ASC of a positively pre-chirped $120 \mathrm{fs}, 100 \mu \mathrm{J}$ pulse at $2 \mu \mathrm{m}$. (a) Pulse evolution as a function of propagation distance. The intensity is shown in log scale. (b) FWHM of a fundamental soliton (blue curve) and FWHM of a compressed pulse (black curve) versus the propagation distance. (c) Compressed pulse (black curve) and transform-limited (TL) pulse (red dashed curve) at HCF output. The fundamental soliton is shown as the blue curve. (d) Optical spectrum (black curve) and soliton spectrum (blue curve) at HCF output.
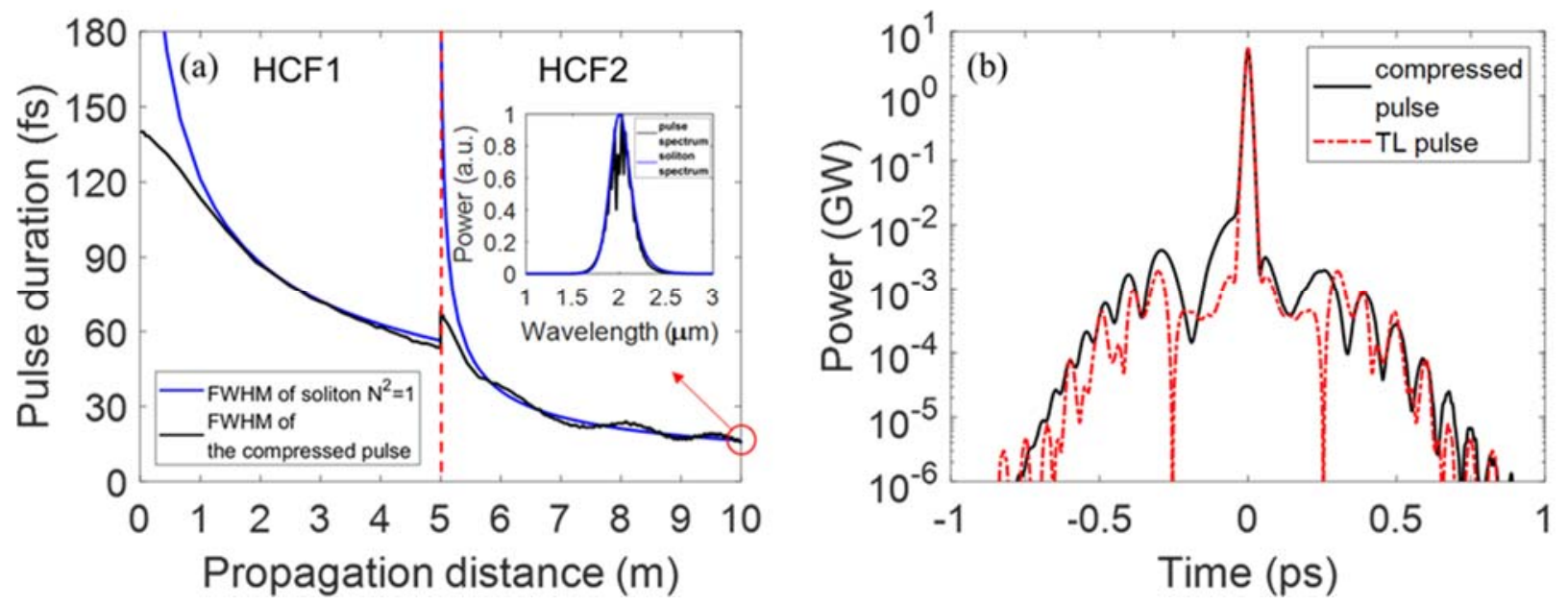

Figure 5. Two-stage ASC of a positively pre-chirped $125 \mu \mathrm{J}$ pulse at $2 \mu \mathrm{m}$. (a) FWHM of a fundamental soliton (blue curve) and FWHM of a compressed pulse (black curve) versus the propagation distance in two-stage ASC. Inset: pulse spectrum (black curve) and soliton spectrum (blue curve) at the output of the second HCF. (b) Compressed pulse (black curve) and transform-limited (TL) pulse (red dashed curve) at HCF output. 
The results in Figure 2 show that ASC is favorable for compressing pulses at a longer center wavelength. To verify this prediction based on analytical analysis, we simulate the propagation of a $280 \mu \mathrm{J}, 220 \mathrm{fs}$ Gaussian pulse ( $240 \mathrm{fs}$ after adding $8000 \mathrm{fs}^{2}$ GDD) centered at $4 \mu \mathrm{m}$ inside an antiresonant HCF with a core radius of $40 \mu \mathrm{m}$. The HCF is filled with helium, with the pressure varying from $p_{0}=1$ bar to $p_{L}=9$ bar. The results in Figure 6 indicate that the pulse can be adiabatically compressed down to $60 \mathrm{fs}$ in duration, corresponding to 4.5 optical cycles. The compressed pulse has a peak power of $4 \mathrm{GW}$, and the pedestal is about $35 \mathrm{~dB}$ below the peak.
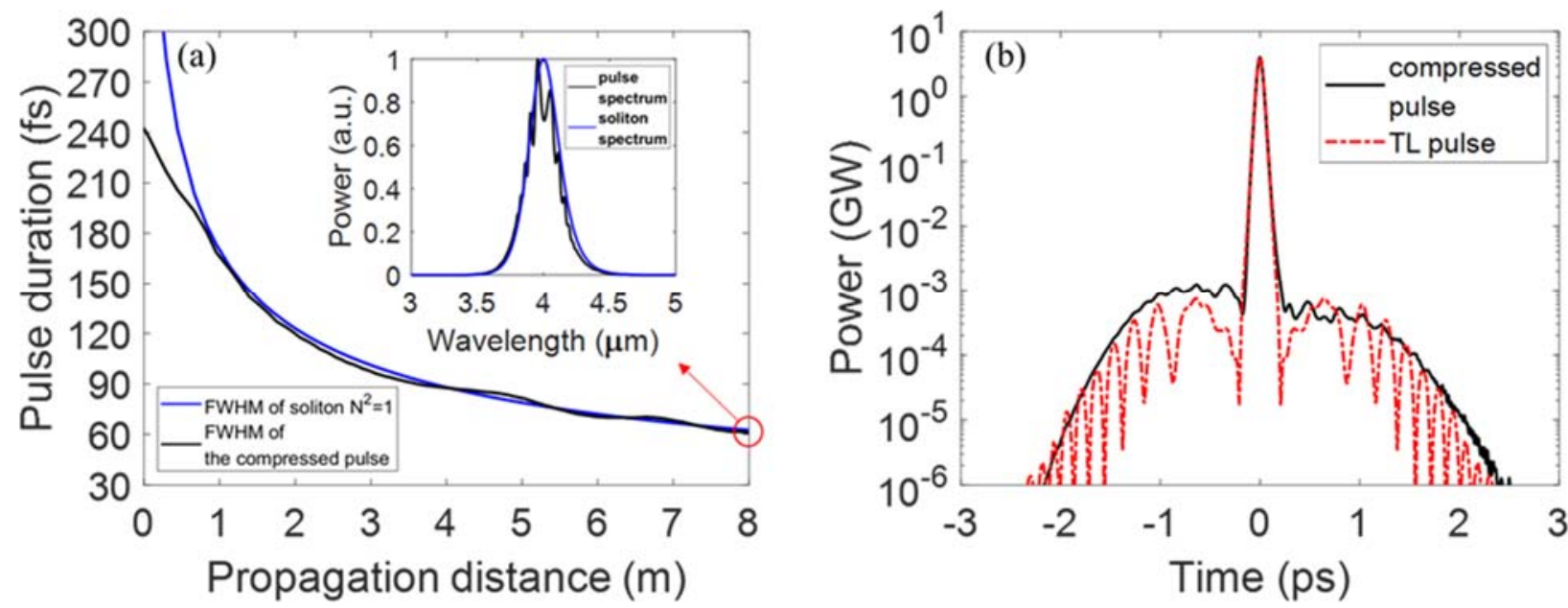

Figure 6. ASC of a positively pre-chirped $280 \mu \mathrm{J}$ pulse at $4 \mu \mathrm{m}$. (a) FWHM of a fundamental soliton (blue curve) and FWHM of ac ompressed pulse (black curve) versus the propagation distance. Inset: pulse spectrum (black curve) and soliton spectrum (blue curve) at the output of the second HCF. (b) Compressed pulse (black curve) and transform-limited (TL) pulse (red dashed curve) at HCF output.

Equations (8) and (9) indicate that increasing the fiber core radius allows compressing pulses with higher energy to a shorter dispersion. We anticipate that energy scaling of ASC to compress $>1 \mathrm{~mJ}$ pulses is possible by using capillary HCFs with the core radius exceeding $100 \mu \mathrm{m}$. In general, ASC requires meters-long propagation distance. Conventional capillary $\mathrm{HCFs}$ that are made of thick-walled rigid fused silica tubes are limited to $\sim 1 \mathrm{~m}$ in length to avoid introducing propagation loss. ASC in this type of HCFs is unlikely possible. In contrast, stretched flexible HCFs (SF-HCFs) that feature much thinner walls (only tens of microns) are being widely used for post-pulse-compression. Thanks to the light weight, these HCFs can be kept straight to prevent excessive loss, even when their length is $6 \mathrm{~m}$ [41]. We expect that pressure-gradient SF-HCFs may allow ASC of $>1 \mathrm{~mJ}$ pulses at $4 \mu \mathrm{m}$, and the resulting few-cycle high-quality pulses constitute an enabling tool for high-field applications.

\section{Conclusions}

In conclusion, we investigated ASC in gas-filled pressure-gradient HCFs. The maximum pulse energy and the minimum compressed pulse duration are dependent on the HCF core size, gas type and pressure, and the center wavelength. We show that properly pre-chirping the pulse prior to compression can accelerate the pulse compression rapidly converging to ASC, which results in improved pulse quality with the pedestal further suppressed. Despite a relatively low compression ratio from single-state ASC, we showed that two-stage ASC can offer a compression ratio of $\sim 9$. As a result, $130 \mathrm{fs}$ pulses at $2 \mu \mathrm{m}$ can be compressed to a nearly two-cycle pulse $15 \mathrm{fs}$ in duration. We also showed that compression of pulses with al onger center wavelength is favored by ASC. For example, a $280 \mu \mathrm{J}, 220 \mathrm{fs}$ Gaussian pulse at $4 \mu \mathrm{m}$ can be compressed to $60 \mathrm{fs}$. Work is ongoing to experimentally verify our theoretical predictions on ASC in gas-filled pressure-gradient HCFs. 
Author Contributions: Conceptualization, R.C. and G.C.; methodology, R.C. and Z.S.; software, R.C. and Z.S.; validation, R.C.; formal analysis, Z.S.; investigation, R.C.; resources, G.C.; data curation, R.C.; writing—original draft preparation, R.C.; writing—review and editing, G.C.; visualization, R.C. and Z.S.; supervision, G.C.; project administration, G.C.; funding acquisition, G.C. All authors have read and agreed to the published version of the manuscript.

Funding: This work is supported by the National Natural Science Foundation of China (no. 11774234), the Guangdong Key R\&D Program (no. 2018B090904003), and the Chinese Academy of Sciences (no. YJKYYQ20190034).

Acknowledgments: We thank Zhiyi Wei of the Institute of Physics CAS for useful discussions.

Conflicts of Interest: The authors declare no conflict of interest.

\section{References}

1. Nagy, T.; Simon, P.; Veisz, L. High-energy few-cycle pulses: Post-compression techniques. Adv. Phys. X 2021, 6, 11845795.

2. Kuehl, H.H. Solitons on an axially nonuniform optical fiber. J. Opt. Soc. Am. B 1988, 5, 709-713. [CrossRef]

3. Smith, K.; Mollenauer, L.F. Experimental-Observation of Adiabatic-Compression and Expansion of Soliton Pulses over Long Fiber Paths. Opt. Lett. 1989, 14, 751-753. [CrossRef]

4. Iwatsuki, K.; Suzuki, K.-I.; Nishi, S. Adiabatic soliton compression of gain-switched DFB-LD pulse by distributed fiber Raman amplification. IEEE Photonics Technol. Lett. 1991, 3, 1074-1076. [CrossRef]

5. Quiroga-Teixeiro, M.L.; Anderson, D.; Andrekson, P.A.; Berntson, A.; Lisak, M. Efficient soliton compression by fast adiabatic amplification. J. Opt. Soc. Am. B 1996, 13, 687-692. [CrossRef]

6. Chernikov, S.V.; Mamyshev, P.V. Femtosecond Soliton Propagation in Fibers with Slowly Decreasing Dispersion. J. Opt. Soc. Am. B 1991, 8, 1633-1641. [CrossRef]

7. Suzuki, K.; Iwatsuki, K.; Nishi, S.; Saruwatari, M.; Kitoh, T. 160 Gbit/s sub-picosecond transform-limited pulse signal generation utilizing adiabatic soliton compression and optical time-division multiplexing. IEEE Photonics Technol. Lett. 1994, 6, 352-354. [CrossRef]

8. Travers, J.C.; Stone, J.M.; Rulkov, A.B.; Cumberland, B.A.; George, A.K.; Popov, S.V.; Knight, J.C.; Taylor, J.R. Optical pulse compression in dispersion decreasing photonic crystal fiber. Opt. Express 2007, 15, 13203-13211. [CrossRef] [PubMed]

9. Tse, M.L.V.; Horak, P.; Price, J.H.V.; Poletti, F.; He, F.; Richardson, D.J. Pulse compression at $1.06 \mu$ m in dispersion-decreasing holey fibers. Opt. Lett. 2006, 31, 3504-3506. [CrossRef] [PubMed]

10. Lenz, G.; Eggleton, B.J. Adiabatic Bragg soliton compression in nonuniform grating structures. J. Opt. Soc. Am. B 1998, 15, 2979-2985. [CrossRef]

11. Russell, P.S.J.; Hölzer, P.; Chang, W.; Abdolvand, A.; Travers, J.C. Hollow-core photonic crystal fibres for gasbased nonlinear optics. Nat. Photonics 2014, 8, 278-286. [CrossRef]

12. Lægsgaard, J.; Roberts, P.J. Theory of adiabatic pressure-gradient soliton compression in hollow-core photonic bandgap fibers. Opt. Lett. 2009, 34, 3710-3712. [CrossRef]

13. Huang, Z.Y.; Chen, Y.F.; Yu, F.; Wu, D.K.; Zhao, Y.; Wang, D.; Leng, Y.X. Ionization-induced adiabatic soliton compression in gas-filled hollow-core photonic crystal fibers. Opt. Lett. 2019, 44, 5562-5565. [CrossRef]

14. Schade, D.; Köttig, F.; Koehler, J.R.; Frosz, M.H.; Russell, P.S.J.; Tani, F. Scaling rules for high quality soliton self-compression in hollow-core fibers. Opt. Express 2021, 29, 19147-19158. [CrossRef] [PubMed]

15. Hasan, M.I.; Akhmediev, N.; Chang, W. Empirical Formulae for Dispersion and Effective Mode Area in Hollow-Core Antiresonant Fibers. J. Lightwave Technol. 2018, 36, 4060-4065. [CrossRef]

16. Nurhuda, M.; Suda, A.; Kaku, M.; Midorikawa, K. Optimization of hollow fiber pulse compression using pressure gradients. Appl. Phys. B 2007, 89, 209-215. [CrossRef]

17. Mak, K.F.; Travers, J.C.; Joly, N.Y.; Abdolvand, A.; Russell, P.S.J. Two techniques for temporal pulse compression in gas-filled hollow-core kagomé photonic crystal fiber. Opt. Lett. 2013, 38, 3592-3595. [CrossRef]

18. Gebhardt, M.; Gaida, C.; Heuermann, T.; Stutzki, F.; Jauregui, C.; Antonio-Lopez, J.; Schulzgen, A.; Amezcua-Correa, R.; Limpert, J.; Tünnermann, A. Nonlinear pulse compression to $43 \mathrm{~W}$ GW-class few-cycle pulses at $2 \mu \mathrm{m}$ wavelength. Opt. Lett. 2017, 42 , 4179-4182. [CrossRef]

19. Vozzi, C.; Nisoli, M.; Sansone, G.; Stagira, S.; Silvestri, S.D. Optimal spectral broadening in hollow-fiber compressor systems. Appl. Phys. B 2005, 80, 285-289. [CrossRef]

20. Wang, P.; Li, Y.; Li, W.; Su, H.; Shao, B.; Li, S.; Wang, C.; Wang, D.; Zhao, R.; Peng, Y.; et al. 2.6 mJ/100 Hz CEP-stable near-single-cycle $4 \mu \mathrm{m}$ laser based on OPCPA and hollow-core fiber compression. Opt. Lett. 2018, 43, 2197-2200. [CrossRef]

21. Kanai, T.; Lee, Y.; Seo, M.; Kim, D.E. Supercontinuum-seeded, carrier-envelope phase-stable, 4. 5-W, 3.8- $\mu$ m, 6-cycle, KTA optical parametric amplifier driven by a 1.4-ps Yb:YAG thin-disk amplifier for nonperturbative spectroscopy in solids. J. Opt. Soc. Am. B 2019, 36, 2407-2413. 
22. Saleh, M.; Chang, W.; Hölzer, P.; Nazarkin, A.; Travers, J.C.; Joly, N.; Russell, P.S.J.; Biancalana, F. Theory of photoionizationinduced blueshift of ultrashort solitons in gas-filled hollow-core photonic crystal fibers. Phys. Rev. Lett. 2011, 107, 203902. [CrossRef] [PubMed]

23. Sprangle, P.; Peñano, J.R.; Hafizi, B. Propagation of intense short laser pulses in the atmosphere. Phys. Rev. E 2002, 66, 046418. [CrossRef] [PubMed]

24. Ammosov, M.V.; Delone, N.B.; Krainov, V.P. Tunnel ionization of complex atoms and of atomic ions in an alternating electromagnetic field. Int. Soc. Opt. Photonics 1986, 64, 1191-1194.

25. Brabec, T.; Krausz, F. Nonlinear Optical Pulse Propagation in the Single-Cycle Regime. Phys. Rev. Lett. 1997, $78,3282-3285$. [CrossRef]

26. Yu, F.; Knight, J. Negative curvature hollow core optical fiber. IEEE J. Sel. Top. Quantum Electron. 2016, 22, 4400610. [CrossRef]

27. Liu, W.; Schimpf, D.N.; Eidam, T.; Limpert, J.; Tuennermann, A.; Kaertner, F.X.; Chang, G.Q. Pre-chirp managed nonlinear amplification in fibers delivering $100 \mathrm{~W}, 60 \mathrm{fs}$ pulse. Opt. Lett. 2015, 40, 151. [CrossRef]

28. Heuermann, T.; Gaida, C.; Gebhardt, M.; Limpert, J. Thulium-doped nonlinear fiber amplifier delivering $50 \mathrm{fs}$ pulses at $20 \mathrm{~W}$ of average power. Opt. Lett. 2018, 43, 4441. [CrossRef]

29. Le, T.; Tempea, G.; Cheng, Z.; Hofer, M.; Stingl, A. Routes to fiber delivery of ultra-short laser pulses in the $25 \mathrm{fs}$ regime. Opt. Lett. 2009, 17, 1240. [CrossRef]

30. Kalashyan, M.; Lefort, C.; -León, L.M.; Mansuryan, T.; Mouradian, L.; Louradour, F. Ultrashort pulse fiber delivery with optimized dispersion control by reflection grisms at $800 \mathrm{~nm}$. Opt. Express 2012, 20, 25624. [CrossRef]

31. Andreana, M.; Le, T.; Drexler, W.; Unterhuber, A. Ultrashort pulse Kagome hollow-core photonic crystal fiber delivery for nonlinear optical imaging. Opt. Lett. 2019, 44, 1588. [CrossRef]

32. Várallyay, Z.; Fekete, J.; Bányász, Á.; Szipőcs, R. Optimizing input and output chirps up to the third-order for sub-nanojoule, ultra-short pulse compression in small core area PCF. Appl. Phys. B 2007, 86, 567. [CrossRef]

33. Martial, I.; Papadopoulos, D.; Hanna, M.; Druon, F.; Georges, P. Nonlinear compression in a rod-type fiber for high energy ultrashort pulse generation. Opt. Express 2009, 17, 11155. [CrossRef] [PubMed]

34. Ganz, T.; Pervak, V.; Apolonski, A.; Baum, P. 16 fs, 350 nJ pulses at 5 MHz repetition rate delivered by chirped pulse compression in fibers. Opt. Lett. 2011, 36, 1107. [CrossRef]

35. Tauser, F.; Adler, F.; Leitenstorfer, A. Widely tunable sub-30-fs pulses from a compact erbium-doped fiber source. Opt. Lett. 2004, 29, 516-518. [CrossRef]

36. Rosenberg, Y.; Drori, J.; Bermudez, D.; Leonhardt, U. Boosting few-cycle soliton self-frequency shift using negative prechirp. Opt. Express 2020, 28, 3107. [CrossRef] [PubMed]

37. Chen, R.; Chang, G. Pre-chirp managed self-phase modulation for efficient generation of wavelength-tunable energetic femtosecond pulses. J. Opt. Soc. Am. B 2020, 37, 2388-2397. [CrossRef]

38. Anderson, D.; Lisak, M.; Malomed, B.; Quiroga-Teixeiro, M. Tunneling of an optical soliton through a fiber junction. J. Opt. Soc. Am. B 1994, 11, 2380-2384. [CrossRef]

39. Bertilsson, K.; Aakjer, T.; Quiroga-Teixeiro, M.L.; Andrekson, P.A.; Hedekvist, P. Investigation of soliton compression by propagation through fiber junctions. Opt. Fiber Technol. 1995, 1, 117-124. [CrossRef]

40. Balciunas, T.; Fourcade-Dutin, C.; Fan, G.; Witting, T.; Voronin, A.A.; Zheltikov, A.M.; Gerome, F.; Paulus, G.G.; Baltuska, A.; Benabid, F. A strong-field driver in the single-cycle regime based on self-compression in a Kagome fibre. Nat. Commun. 2015, 6, 6117. [CrossRef] [PubMed]

41. Nagy, T.; Hädrich, S.; Simon, P.; Blumenstein, A.; Walther, N.; Klas, R.; Buldt, J.; Stark, H.; Breitkopf, S.; Jójárt, P.; et al. Generation of three-cycle multi-millijoule laser pulses at $318 \mathrm{~W}$ average power. Optica 2019, 6, 1423-1424. [CrossRef] 